\title{
ON THE GEODETIC HULL NUMBER FOR COMPLEMENTARY PRISMS II
}

\author{
Diane Castonguay, Erika M. M. Coelho, Hebert Coelho and \\ Julliano R. NAscimento*
}

\begin{abstract}
In the geodetic convexity, a set of vertices $S$ of a graph $G$ is convex if all vertices belonging to any shortest path between two vertices of $S$ lie in $S$. The convex hull $H(S)$ of $S$ is the smallest convex set containing $S$. If $H(S)=V(G)$, then $S$ is a hull set. The cardinality $h(G)$ of a minimum hull set of $G$ is the hull number of $G$. The complementary prism $G \bar{G}$ of a graph $G$ arises from the disjoint union of the graph $G$ and $\bar{G}$ by adding the edges of a perfect matching between the corresponding vertices of $G$ and $\bar{G}$. A graph $G$ is autoconnected if both $G$ and $\bar{G}$ are connected. Motivated by previous work, we study the hull number for complementary prisms of autoconnected graphs. When $G$ is a split graph, we present lower and upper bounds showing that the hull number is unlimited. In the other case, when $G$ is a non-split graph, it is limited by 3 .
\end{abstract}

Mathematics Subject Classification. 05C12, 05C76.

Received May 13, 2020. Accepted August 20, 2020.

\section{INTRODUCTION}

For a finite and simple graph $G$ with vertex set $V(G)$, a graph convexity on $V(G)$ is a collection $\mathcal{C}$ of subsets of $V(G)$ such that $\emptyset, V(G) \in \mathcal{C}$ and $\mathcal{C}$ is closed under intersections. The sets in $\mathcal{C}$ are called convex sets and the convex hull $H_{\mathcal{C}}(S)$ in $\mathcal{C}$ of a set $S$ of vertices of $G$ is the smallest set in $\mathcal{C}$ containing $S$. Some natural convexities in graphs are defined by a set $\mathcal{P}$ of paths in $G$, in a way that a set $S$ of vertices of $G$ is convex if and only if for every path $P=v_{0} v_{1} \ldots v_{l}$ in $\mathcal{P}$ such that if $v_{0}$ and $v_{l}$ belong to $S$, then all vertices of $P$ belong to $S$.

The definition of convex sets in graphs originally come from Euclidean geometry, in which a set $S$ is convex if every line segment between two points of $S$ remains in $S$. The concepts of convexity in graphs can be applied to model contexts involving some disseminating processes between entities, e.g. marketing strategies [7], spread of disease and opinion [13], and distributed computing [20].

In this paper we study the convexity related to shortest paths in graphs, the geodetic convexity $\mathcal{C}$. Given a graph $G$, the closed interval $I[u, v]$ of a pair $u, v \in V(G)$ consists of all vertices lying in any shortest $(u, v)$ path in $G$. For a set $S \subseteq V(G)$, the closed interval $I[S]$ is the union of all sets $I[u, v]$ for $u, v \in S$. If $I[S]=S$, then $S$ is a convex set. The convex hull $H_{\mathcal{C}}(S)$ of $S$ is the smallest convex set containing $S$. Since a graph $G$ uniquely determines its geodetic convexity $\mathcal{C}$, we may write $H(S)$, instead of $H_{\mathcal{C}}(S)$. If $H(S)=V(G)$ we say that $S$ is a hull set of $G$. The cardinality $h(G)$ of a minimum hull set of $G$ is called the (geodetic) hull number of $G$.

Keywords. Geodetic convexity, hull set, hull number, complementary prism.

INF, Universidade Federal de Goiás, Goiânia, Brazil.

*Corresponding author: julliano.rn@gmail.com, julliano@inf.ufg.br 
Everett and Seidman [15] introduced the concept of hull number in the geodetic convexity. For some later results see, e.g. [3,10,18]. The computation of hull number is NP-hard for bipartite graphs [3], partial cubes [1], and $P_{9}$-free graphs [12], but it can be computed in polynomial time for cographs, split graphs [9], $(q, q-4)$-graphs [3], \{paw, $\left.P_{5}\right\}$-free graphs [12], and distance-hereditary graphs [19].

The hull number is also studied in other graph convexities. It can be determined in polynomial time for general graphs in triangle path convexity [8], related to paths which allow only short chords, and monophonic convexity [11], related to induced paths. In $P_{3}$-convexity, that considers paths with three vertices, computing the hull number for general graphs is NP-complete [5]. However, Duarte et al. [14] showed that the hull number of complementary prisms can be determined efficiently in $P_{3}$-convexity, more specifically, it is limited by 5 when $G$ and $\bar{G}$ are connected.

In 2007, Haynes et al. [17] introduced the complementary product, an operation that generalizes the Cartesian product of two graphs. In their same work, they introduce a particular case of complementary product called complementary prism. The complementary prism $G \bar{G}$ of a graph $G$ arises from the disjoint union of the graph $G$ and its complement $\bar{G}$ by adding the edges of a perfect matching between vertices with the same label in $G$ and $\bar{G}$. Related to geodetic convexity, we mention that some works were conducted for graph operations like join, composition, and Cartesian product [4], lexicographic product [2], and strong product [21].

Considering the geodetic hull number in complementary prisms $G \bar{G}$, in our previous paper [6], we presented bounds when $G$ is a disconnected graph or $G$ is a tree. Here, we present lower and upper bounds on the hull number of complementary prisms $G \bar{G}$ when both $G$ and $\bar{G}$ are connected. In particular, we show that $h(G \bar{G}) \leq 3$ when $G$ is a non-split graph. Otherwise, we characterize convex sets in split graphs $G$ that allow the hull number for $G \bar{G}$ be unlimited.

This paper is divided into three more sections. In Section 2, we define the fundamental concepts. In Section 3 , we present our main results.

\section{Preliminaries}

Before we discuss our contributions, we present some relevant definitions. All graphs will be finite, simple, and undirected, and we use standard terminology and notation.

Let $G$ be a graph. Given a vertex $v \in V(G)$, its open neighborhood is denoted by $N_{G}(v)$, and its closed neighborhood, denoted by $N_{G}[v]$, is the set $N_{G}[v]=N_{G}(v) \cup\{v\}$. For a set $U \subseteq V(G)$, let $N_{G}(U)=\bigcup_{v \in U} N_{G}(v) \backslash U$, and $N_{G}[U]=N_{G}(U) \cup U$. We denote the degree of a vertex $v \in V(G)$ by $\operatorname{deg}_{G}(v)$. If $\operatorname{deg}_{G}(v)=0$, then we say that $v$ is an isolated vertex.

A $K_{n}$ (resp. $C_{n}$ ) in a graph $G$ denotes an induced complete subgraph (resp. cycle) on $n$ vertices. A clique (resp. independent set) is a set of pairwise adjacent (resp. non-adjacent) vertices. A vertex of a graph $G$ is simplicial in $G$ if its neighborhood is a clique.

The distance $d_{G}(u, v)$ of two vertices $u$ and $v$ in $G$ is the minimum number of edges of a path in $G$ between $u$ and $v$. The greatest distance between any two vertices in $G$ is the diameter of $G$, denoted by $\operatorname{diam}(G)$. We say that $H$ is an isometric subgraph of $G$ if $H$ is a subgraph of $G$ such that $d_{H}(u, v)=d_{G}(u, v)$ for any pair $u, v \in V(H)$.

A graph $G$ is called connected if any two of its vertices are linked by a path in $G$. Otherwise, $G$ is called disconnected. A maximal connected subgraph of $G$ is called a connected component or component of $G$. A component $G_{i}$ of a graph $G$ is trivial if $\left|V\left(G_{i}\right)\right|=1$, and non-trivial otherwise. If a graph $G$ is connected and its complement $\bar{G}$ is also connected, we say that $G$ is an autoconnected graph. Let $G$ be a graph and $\bar{G}$ its complement. For every vertex $v \in V(G)$, we denote $\bar{v} \in V(\bar{G})$ as its corresponding vertex, and for a set $X \subseteq V(G)$, we let $\bar{X}$ be the corresponding set of vertices in $V(\bar{G})$. The set of positive integers $\{1, \ldots, k\}$ is denoted by $[k]$.

For a graph $G$ with vertex set $V(G)=\left\{v_{1}, \ldots, v_{n}\right\}$ and edge set $E(G)$, the complementary prism of $G$ is the graph denoted by $G \bar{G}$ with vertex set $V(G \bar{G})=\left\{v_{1}, \ldots, v_{n}\right\} \cup\left\{\bar{v}_{1}, \ldots, \bar{v}_{n}\right\}$ and edge set $E(G \bar{G})=E(G) \cup\left\{\bar{v}_{i} \bar{v}_{j}\right.$ : $1 \leq i<j \leq n$ and $\left.v_{i} v_{j} \notin E(G)\right\} \cup\left\{v_{1} \bar{v}_{1}, \ldots, v_{n} \bar{v}_{n}\right\}$. Let $S \subseteq V(G \bar{G})$. Throughout this paper we consider 
the convex hull $H(S)$ most of times on the graph $G \bar{G}$. If we need to indicate the convex hull on another graph, say $G$, we add a subscript to the notation, e.g. $H_{G}(S)$.

A split graph $G$ is one whose vertex set admits a partition $V(G)=C \cup I$ into a clique $C$ and an independent set $I$. If $G$ is a split graph, we consider the partition of $V(G)$ such that $C$ is a maximum clique.

\section{RESULTS}

\subsection{General and non-split graphs}

We begin by showing three fundamental lemmas and a proposition that we use in the sequel.

Lemma 3.1 (Dourado et al. [9]). Let $G$ be a graph and $S$ a proper and non-empty subset of $V(G)$. If $V(G) \backslash S$ is convex then every hull set of $G$ contains at least one vertex of $S$.

Lemma 3.2 (Dourado et al. [9]). Let $G$ be a graph and $H$ an isometric subgraph of $G$. Then for every hull set $S$ of $H$ it holds that $V(H) \subseteq H_{G}(S)$.

Lemma 3.3 (Coelho et al. [6]). Let $G$ be a graph. If $u$ is a simplicial vertex in $G$ and $\bar{u}$ is a simplicial vertex in $\bar{G}$, then every hull set $S$ of $G \bar{G}$ intersects $\{u, \bar{u}\}$.

Proposition 3.4. Let $G$ be a graph, $S \subseteq V(G \bar{G})$, and $v_{1} \ldots v_{k}$ be a path in $G$, for $k \geq 2$. If $\left\{v_{1}, \bar{v}_{2}, \ldots, \bar{v}_{k}\right\} \subseteq$ $H(S)$, then $v_{k} \in H(S)$.

Proof. The proof is by induction on $k$. First, let $k=2$. Since $v_{1} v_{2} \in E(G)$ and $v_{1}, \bar{v}_{2} \in H(S), v_{2} \in I\left[v_{1}, \bar{v}_{2}\right]$. Now, let $k>2$. Let $v_{1} \ldots v_{k-1} v_{k}$ be a path in $G$ and suppose that $\left\{v_{1}, \bar{v}_{2}, \ldots, \bar{v}_{k-1}, \bar{v}_{k}\right\} \subseteq H(S)$. By induction hypothesis $v_{k-1} \in H(S)$, which implies that $v_{k} \in I\left[v_{k-1}, \bar{v}_{k}\right]$. Therefore, it follows that $v_{k} \in H(S)$, for $k \geq 2$.

Next we state our contributions. We first show that for complementary prisms of non-split graphs the geodetic hull number is limited by three.

Theorem 3.5. Let $G$ be a non-split autoconnected graph. Then $h(G \bar{G}) \leq 3$.

Proof. Suppose that $G$ is a non-split graph. According to Foldes and Hammer [16], $G$ is split if and only if $G$ does not have an induced subgraph isomorphic to one of the three forbidden graphs, $C_{4}, C_{5}$, or $2 K_{2}$. To show the upper bound $h(G \bar{G}) \leq 3$ we construct hull sets on three cases: (1) $C_{4}$ or (2) $C_{5}$ or (3) $2 K_{2}$ are induced subgraphs of $G$.

Case 1. Suppose that $C_{4}$ is an induced subgraph of $G$.

Let $V\left(C_{4}\right)=\left\{u_{1}, \ldots, u_{4}\right\}, E\left(C_{4}\right)=\left\{u_{i} u_{i+1}: 1 \leq i \leq 3\right\} \cup\left\{u_{4} u_{1}\right\}$, and $S=\left\{u_{1}, \bar{u}_{3}, \bar{u}_{4}\right\}$. We show that $H(S)=V(G \bar{G})$.

First, we show that $V\left(C_{4}\right) \cup \overline{V\left(C_{4}\right)} \subseteq H(S)$. We have that $u_{4} \in I\left[u_{1}, \bar{u}_{4}\right]$ and $\bar{u}_{1} \in I\left[u_{1}, \bar{u}_{3}\right]$. Consequently $u_{3} \in I\left[u_{4}, \bar{u}_{3}\right]$ and $u_{2} \in I\left[u_{1}, u_{3}\right]$, then $\bar{u}_{2} \in I\left[u_{2}, \bar{u}_{4}\right]$. Hence $V\left(C_{4}\right) \cup \overline{V\left(C_{4}\right)} \subseteq H(S)$.

Let $A=\left(N_{G}\left(u_{1}\right) \cap N_{G}\left(u_{3}\right)\right) \cup\left(N_{G}\left(u_{2}\right) \cap N_{G}\left(u_{4}\right)\right)$ and $v \in A$. Since $u_{1} u_{3} \notin E(G)$ (resp. $\left.u_{2} u_{4} \notin E(G)\right)$, then $v \in I\left[u_{1}, u_{3}\right]$ (resp. $v \in I\left[u_{2}, u_{4}\right]$ ), hence $A \subseteq H(S)$.

Let $B=V(G) \backslash\left(A \cup V\left(C_{4}\right)\right)$. For every $b \in B$, there exist $i, j \in[4], i \neq j$, such that $u_{i} u_{j} \in E(G)$ and $b \notin N_{G}\left(\left\{u_{i}, u_{j}\right\}\right)$. Then $\bar{u}_{i} \bar{u}_{j} \notin E(\bar{G})$, consequently $\bar{b} \in I\left[\bar{u}_{i} \bar{u}_{j}\right]$, hence $\bar{B} \subseteq H(S)$.

Since $G$ is connected, there exists a path in $G$ joining every vertex from $B$ to a vertex in $A \cup V\left(C_{4}\right)$. Since $\bar{B} \subseteq H(S)$, then Proposition 3.4 implies that $B \subseteq H(S)$. Hence $V(G) \subseteq H(S)$. Since $\bar{G}$ is connected, also by Proposition 3.4, $\bar{A} \subseteq H(S)$. Therefore $S$ is a hull set of $G \bar{G}$.

Case 2. Suppose that $C_{5}$ is an induced subgraph of $G$. 


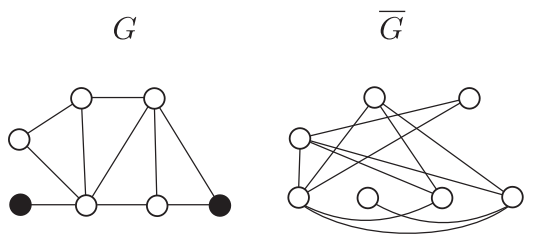

(a)
$G \quad \bar{G}$

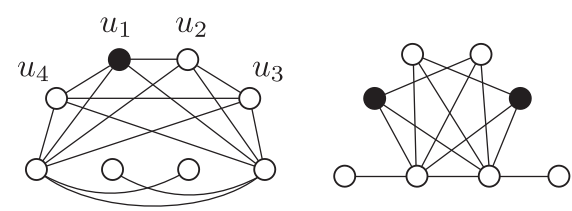

(b)

FIGURE 1. Graphs satisfying $h(G \bar{G}) \leq 3$. (a) $h(G \bar{G})=2$. (b) $h(G \bar{G})=3$.

Let $V\left(C_{5}\right)=\left\{u_{1}, \ldots, u_{5}\right\}, E\left(C_{5}\right)=\left\{u_{i} u_{i+1}: 1 \leq i \leq 4\right\} \cup\left\{u_{5} u_{1}\right\}$, and $S=\left\{u_{1}, u_{4}, \bar{u}_{3}\right\}$. We show that $H(S)=V(G \bar{G})$.

First, we show that $V\left(C_{5}\right) \cup \overline{V\left(C_{5}\right)} \subseteq H(S)$. We have that $u_{5} \in I\left[u_{1}, u_{4}\right], \bar{u}_{1} \in I\left[u_{1}, \bar{u}_{3}\right]$ and $u_{3} \in I\left[u_{4}, \bar{u}_{3}\right]$. Then $u_{2} \in I\left[u_{1}, u_{3}\right]$. By Proposition 3.4 (dual), we have that $\bar{u}_{5}, \bar{u}_{4}, \bar{u}_{2} \in H(S)$. Hence $V\left(C_{5}\right) \cup \overline{V\left(C_{5}\right)} \subseteq H(S)$.

Let

$$
A=\bigcup_{i, j \in[5], i \neq j: u_{i} u_{j} \notin E(G)}\left(N_{G}\left(u_{i}\right) \cap N_{G}\left(u_{j}\right)\right) .
$$

By the definition of $A$, for every $v \in A$, there exists $i, j \in[5], i \neq j$, such that $u_{i} u_{j} \notin E(G)$, which implies that $v \in I\left[u_{i}, u_{j}\right]$. Hence $A \subseteq H(S)$.

Now, let $B=V(G) \backslash\left(A \cup V\left(C_{5}\right)\right)$. By the definition of $A$ is $b$ a neighbor of at most two vertices from $C_{5}$ in $G$. Therefore is $\bar{b}$ a neighbor of at least three vertices from $\overline{C_{5}}$. Moreover, at least two of the mentioned three vertices are not adjacent. Clearly, $\bar{b}$ is a common neighbor of both of them and we have $\bar{b} \in H(S)$. Hence $\bar{B} \subseteq H(S)$.

Since $G$ is connected, there exists a path in $G$ joining every vertex from $B$ to a vertex in $A \cup V\left(C_{5}\right)$. Since $\bar{B} \subseteq H(S)$, then Proposition 3.4 implies that $B \subseteq H(S)$. Hence $V(G) \subseteq H(S)$. Since $\bar{G}$ is connected, also by Proposition 3.4, $\bar{A} \subseteq H(S)$. Therefore $S$ is a hull set of $G \bar{G}$.

Case 3. Suppose that $2 K_{2}$ is an induced subgraph of $G$.

Since $2 K_{2}$ (resp. $G \bar{G}$ ) is isomorphic to $\bar{C}_{4}$ (resp. $\bar{G} G$ ), we consider $\bar{G} G$ and the proof follows by Case 1 .

Since there exists a hull set of order three in all cases the upper bound $h(G \bar{G}) \leq 3$ holds for a non-split graph $G$.

For an illustration of the bound of Theorem 3.5 see Figure 1b. The black vertices represent a hull set of each complementary prism $G \bar{G}$. For convenience, the edges joining corresponding vertices from $G$ to $\bar{G}$ are not depicted in the figure.

We show a lower bound by restricting the diameter of the graphs $G$ and $\bar{G}$. That result follows in Theorem 3.6.

Theorem 3.6. Let $G$ be an autoconnected graph. If $\operatorname{diam}(G)=\operatorname{diam}(\bar{G})=2$, then $h(G \bar{G}) \geq 3$.

Proof. For contradiction, suppose that $h(G \bar{G})<3$. We show that every set $S \subseteq V(G \bar{G})$ of order 2 is not a hull set of $G \bar{G}$. First, let $u, v \in V(G)$. Consider $S_{1}=\{u, v\}$, and $S_{2}=\{\bar{u}, \bar{v}\}$.

Since $\operatorname{diam}(G)=\operatorname{diam}(\bar{G})=2, H\left(S_{1}\right) \cap V(\bar{G})=\emptyset\left(\right.$ resp. $\left.H\left(S_{2}\right) \cap V(G)=\emptyset\right)$, then $S_{1}$ (resp. $\left.S_{2}\right)$ is not a hull set of $G \bar{G}$.

Now, let $u \in V(G), \bar{v} \in V(\bar{G})$, and $S_{3}=\{u, \bar{v}\}$. If $u v \in E(G)$ (resp. $u v \notin E(G)$ ), then $H\left(S_{3}\right)=\{u, v, \bar{v}\}$ (resp. $\left.H\left(S_{3}\right)=\{u, \bar{u}, \bar{v}\}\right)$. Since $\bar{u} \notin H\left(S_{3}\right)$ (resp. $v \notin H\left(S_{3}\right)$ ), then $S_{3}$ is not a hull set of $G \bar{G}$.

The two previous results imply in Corollary 3.7. 


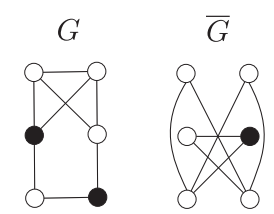

Figure 2. Non-split graph $G$ with $\operatorname{diam}(G)=\operatorname{diam}(\bar{G})=2$.

Corollary 3.7. Let $G$ be a non-split autoconnected graph. If $\operatorname{diam}(G)=\operatorname{diam}(\bar{G})=2$, then $h(G \bar{G})=3$.

Proof. It follows directly from Theorems 3.5 and 3.6.

For an illustration of Corollary 3.7 see in Figure 2 an example of non-split autoconnected graph $G$ with $\operatorname{diam}(G)=\operatorname{diam}(\bar{G})=2$ and $h(G \bar{G})=3$. The black vertices represent a hull set of $G \bar{G}$.

We consider in Theorem 3.8 graphs $G$ with diameter greater than three. Notice that the condition $\operatorname{diam}(G)>3$ implies that $G$ is not a split graph.

Theorem 3.8. Let $G$ be an autoconnected graph. If $\operatorname{diam}(G)>3$, then $h(G \bar{G})=2$.

Proof. Since $\operatorname{diam}(G)>3$, there exist at least two vertices $x, y \in V(G)$ such that $d_{G}(x, y)>3$. Thus, we can define an induced path $P=u_{1} u_{2} u_{3} u_{4} u_{5}$ in $G$ such that $d_{G}\left(u_{1}, u_{5}\right)=4$. Let $S=\left\{u_{1}, u_{4}\right\}$. We show that $S$ is a hull set of $G \bar{G}$.

Let $U=\left\{u_{1}, u_{2}, u_{3}, u_{4}\right\}$. Since $u_{1} u_{2} u_{3} u_{4}$, and $u_{1} \bar{u}_{1} \bar{u}_{4} u_{4}$ are shortest $\left(u_{1}, u_{4}\right)$-paths, we have that $U \cup$ $\left\{\bar{u}_{1}, \bar{u}_{4}\right\} \subseteq I[S]$. Consequently $\bar{u}_{2} \in I\left[u_{2}, \bar{u}_{4}\right]$, and $\bar{u}_{3} \in I\left[u_{3}, \bar{u}_{1}\right]$, which implies that $U \cup \bar{U} \subseteq H(S)$.

To complete the proof, we show that for every vertex $z \in V(G) \backslash U$, its corresponding vertex $\bar{z}$ belongs to $H(S)$. In view of the number of neighbors that $z$ has in $U$, we deal with three cases. Notice that, since $d_{G \bar{G}}\left(u_{1}, u_{4}\right)=3, z$ is not adjacent to both $u_{1}$ and $u_{4}$.

Case 1. $\left|N_{G}(z) \cap U\right| \leq 1$.

In this case, $\bar{z}$ is neighbor of two non-adjacent vertices in $\left\{\bar{u}_{1}, \bar{u}_{2}, \bar{u}_{3}, \bar{u}_{4}\right\}$, say $\bar{u}_{i}$ and $\bar{u}_{j}$, for $i, j \in[4], i \neq j$. Then $\bar{z} \in I\left[\bar{u}_{i}, \bar{u}_{j}\right]$. Notice that by this case we obtain that $\bar{u}_{5} \in I\left[\bar{u}_{1}, \bar{u}_{2}\right]$ and also $u_{5} \in I\left[u_{4}, \bar{u}_{5}\right]$.

Case 2. $\left|N_{G}(z) \cap U\right|=2$.

Suppose that $z$ has two neighbors $u_{i}, u_{j} \in U$, for $i, j \in[4], i \neq j$. If $i, j \in\{1,2\}$ (resp. $i, j \in\{3,4\}$ ), $i \neq j$, we fall back on Case 1. If $i, j \in\{2,3\}, i \neq j$, then $\bar{z} \in I\left[\bar{u}_{4}, \bar{u}_{5}\right]$. If $i, j \in\{1,3\}$, (resp. $\left.i, j \in\{2,4\}\right) i \neq j$, we obtain that $z \in I\left[u_{1}, u_{4}\right]$. Since $z \in H(S)$, then $\bar{z} \in I\left[z, \bar{u}_{k}\right]$, for $k=2$ (resp. $k=3$ ).

Case 3. $\left|N_{G}(z) \cap U\right|=3$.

Suppose that $z$ has three neighbors $u_{i}, u_{j}, u_{k} \in U$, for $i, j, k \in[4], i \neq j \neq k$. Since $d_{G \bar{G}}\left(u_{1}, u_{4}\right)=3$, $\{i, j, k\}=\{1,2,3\}$ or $\{2,3,4\}$. Then, we have that $z \in I\left[u_{1}, u_{4}\right]$. Since $z \in H(S)$, then $\bar{z} \in I\left[z, \bar{u}_{k}\right]$, for $k=4$ or $k=1$.

By all cases, we conclude that $z \in V(G) \backslash U, \bar{z} \in H(S)$. This way, we have that $V(\bar{G}) \subseteq H(S)$. Since $G$ is connected, and $U \cup V(\bar{G}) \subseteq H(S)$, Proposition 3.4 implies that $V(G) \subseteq H(S)$. Therefore $S$ is a hull set of $G \bar{G}$, and $h(G \bar{G})=2$, which completes the proof. 


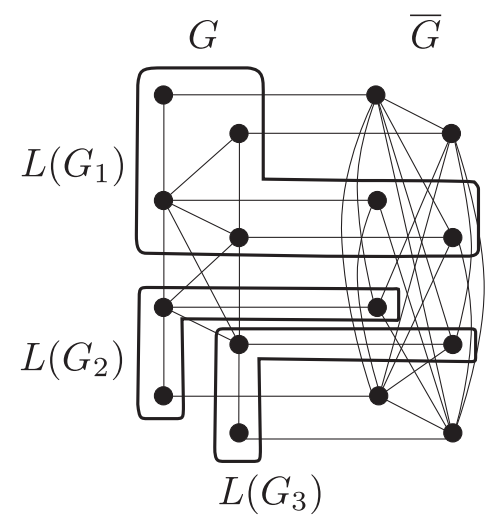

Figure 3 . Sets $L\left(G_{i}\right)$ of $G^{\prime}$ on the graph $G \bar{G}$, for $i \in[3]$.

\subsection{Split graphs}

The following definitions and lemma characterize some convex sets in split graphs.

Let $G=(C \cup I, E)$ be a split graph. Let the graph $G^{\prime}$ arise from $G$ by removing the edges of the graph induced by $C$. We call the graph $G^{\prime}$ as the component graph of $G$. We denote by $c\left(G^{\prime}\right)$ the number of connected components of $G^{\prime}$. We denote by $G_{i}, 1 \leq i \leq c\left(G^{\prime}\right)$, the connected components of $G^{\prime}$. Furthermore, we refer to $n t\left(G^{\prime}\right)$ and $t\left(G^{\prime}\right)$ as the number of non-trivial and trivial components of $G^{\prime}$, respectively. Definition 3.9 expresses formally the idea of a component graph.

Definition 3.9. Let $G=(C \cup I, E)$ be a split graph. We define the component graph $G^{\prime}$ of $G$ as

$$
G^{\prime}=G \backslash E(G[C])=\bigcup_{i=1}^{c\left(G^{\prime}\right)} G_{i} .
$$

Definition 3.10. Let $G=(C \cup I, E)$ be a split autoconnected graph. We define the set $L\left(G_{i}\right)$ of a component $G_{i}$ of $G^{\prime}$ as

$$
L\left(G_{i}\right)=V\left(G_{i}\right) \cup\left(V\left(\bar{G}_{i}\right) \cap \bar{C}\right),
$$

for every $i \in\left[c\left(G^{\prime}\right)\right]$.

See in Figure 3 examples of sets $L\left(G_{i}\right)$ of $G^{\prime}$, for $i \in[3]$.

Lemma 3.11. The set $V(G \bar{G}) \backslash L\left(G_{i}\right)$ is convex in $G \bar{G}$, for every $i \in\left[c\left(G^{\prime}\right)\right]$.

Proof. Let $i \in\left[c\left(G^{\prime}\right)\right]$. We show that the closed interval of any two vertices of $V(G \bar{G}) \backslash L\left(G_{i}\right)$ does not intersect $L\left(G_{i}\right)$. We have three cases:

(1) $w, w^{\prime} \in V(G) \backslash V\left(G_{i}\right)$;

(2) $\bar{w}, \bar{w}^{\prime} \in V(\bar{G}) \backslash\left(V\left(\bar{G}_{i}\right) \cap \bar{C}\right)$;

(3) $w \in V(G) \backslash V\left(G_{i}\right)$ and $\bar{w}^{\prime} \in V(\bar{G}) \backslash\left(V\left(\bar{G}_{i}\right) \cap \bar{C}\right)$.

Case 1. Let $w, w^{\prime} \in V(G) \backslash V\left(G_{i}\right)$, and $v \in V\left(G_{i}\right)$.

Let $P$ be a shortest $\left(w, w^{\prime}\right)$-path passing through $v$. Since $G$ is a split graph and by definition of $G_{i}, P$ contain two vertices $x, y \in C$. Then $x y \in E(G)$ and thus $P$ cannot be a shortest path, a contradiction.

Case 2. Let $\bar{w}, \bar{w}^{\prime} \in V(\bar{G}) \backslash\left(V\left(\bar{G}_{i}\right) \cap \bar{C}\right)$, and $v \in L\left(G_{i}\right)$. 
Let $P$ be a shortest $\left(\bar{w}, \bar{w}^{\prime}\right)$-path passing through $v$. Clearly, $\bar{w} \bar{w}^{\prime} \notin E(\bar{G})$ and therefore $w w^{\prime} \in E(G)$.

If $v \in V\left(G_{i}\right)$, this implies that $v=w$ or $v=w^{\prime}$, but this yields a contradiction since both $w, w^{\prime}$ cannot belong to $I$ and one of $w, w^{\prime}$ must belong to $V\left(G_{i}\right) \cap C$. If $v \in V\left(\bar{G}_{i}\right) \cap \bar{C}, N_{\bar{G}}(v)$ is a clique, and thus $P$ cannot be a shortest $\left(\bar{w}, \bar{w}^{\prime}\right)$-path, a contradiction.

Case 3. Let $w \in V(G) \backslash V\left(G_{i}\right)$, and $\bar{w}^{\prime} \in V(\bar{G}) \backslash\left(V\left(\bar{G}_{i}\right) \cap \bar{C}\right)$.

If $\bar{w}^{\prime}=\bar{w}$, then $I\left[w, \bar{w}^{\prime}\right]=\left\{w, \bar{w}^{\prime}\right\}$. Now suppose that $\bar{w}^{\prime} \neq \bar{w}$. If $w w^{\prime} \notin E(G)$, then $I\left[w, \bar{w}^{\prime}\right]=\left\{w, \bar{w}^{\prime}, \bar{w}\right\}$. Otherwise, $w w^{\prime} \in E(G)$, then $I\left[w, \bar{w}^{\prime}\right]=\left\{w, \bar{w}^{\prime}, w^{\prime}\right\}$. Hence $I\left[w, \bar{w}^{\prime}\right] \cap L\left(G_{i}\right)=\emptyset$.

By all cases, we obtain that for all $w, w^{\prime} \in V(G \bar{G}) \backslash L\left(G_{i}\right), I\left[w, w^{\prime}\right] \cap L\left(G_{i}\right)=\emptyset$. Therefore, the set $V(G \bar{G}) \backslash$ $L\left(G_{i}\right)$ is convex in $G \bar{G}$, for every $i \in\left[c\left(G^{\prime}\right)\right]$.

Since $\bar{G}$ is also split, Lemma 3.11 holds for $\bar{G} G$, that is, considering $\bigcup_{j=1}^{c\left(\bar{G}^{\prime}\right)} G_{j}$ be the components of $\bar{G}^{\prime}$, we have that the set $V(\bar{G} G) \backslash L\left(\bar{G}_{j}\right)$ is convex in $\bar{G} G$, for every $j \in\left[c\left(\bar{G}^{\prime}\right)\right]$. In view of that, we achieve the lower bound result.

Theorem 3.12. Let $G$ be a split autoconnected graph. It holds that $h(G \bar{G}) \geq \max \left\{c\left(G^{\prime}\right), c\left(\bar{G}^{\prime}\right), 2\right\}$.

Proof. By Lemma 3.11, $V(G \bar{G}) \backslash L\left(G_{i}\right)$, for every $i \in\left[c\left(G^{\prime}\right)\right]$, and $V(G \bar{G}) \backslash L\left(\bar{G}_{j}\right)$, for every $j \in\left[c\left(\bar{G}^{\prime}\right)\right]$, are convex sets. Thus, Lemma 3.1 implies that every hull set of $G \bar{G}$ must contain at least one vertex from $L\left(G_{i}\right)$ and $L\left(\bar{G}_{j}\right)$. Since the components $G_{i}$ and $G_{i^{\prime}}$, (resp. $G_{j}$ and $\left.G_{j^{\prime}}\right)$ are disjoints for all $i, i^{\prime} \in\left[c\left(G^{\prime}\right)\right]$, $i \neq i^{\prime}$, (resp. $\left.j, j^{\prime} \in\left[c\left(\bar{G}^{\prime}\right)\right], j \neq j^{\prime}\right)$, each vertex $v \in V(G)$ (resp. $\bar{v} \in V(\bar{G})$ ) intersects exactly one $L\left(G_{i}\right)$ (resp. $L\left(G_{j}\right)$ ). This implies that $h(G \bar{G}) \geq \max \left\{c\left(G^{\prime}\right), c\left(\bar{G}^{\prime}\right)\right\}$. Since $|V(G)| \geq 2, h(G \bar{G}) \geq 2$, hence the result $h(G \bar{G}) \geq \max \left\{c\left(G^{\prime}\right), c\left(\bar{G}^{\prime}\right), 2\right\}$ holds.

Proceeding on the upper bound results, first we present some useful lemmas. Let $S$ be a subset of the vertices of a graph $G$. In Lemma 3.13 we show that for two vertices $u, v$ in $V(G)$ at distance three from each other, if $u, v \in H(S)$, then their closed neighborhoods $N_{G}[\{u, v\}]$ as well as $\overline{N_{G}[\{u, v\}]}$ belong to $H(S)$. Furthermore, we deal with a split graph $G$ that has only one trivial component in $G^{\prime}$. In Lemma 3.14 we show that if $G^{\prime}$ has only one trivial component, then $\bar{G}^{\prime}$ also has only one trivial component, and vice versa.

Lemma 3.13. Let $G=(C \cup I, E)$ be a split autoconnected graph, $u, v \in I$ and $S \subseteq V(G \bar{G})$. If $d_{G}(u, v)=3$ and $u, v \in H(S)$, then $N_{G}[\{u, v\}] \cup \overline{N_{G}[\{u, v\}]} \subseteq H(S)$.

Proof. Let $u, v \in I$ such that $d_{G}(u, v)=3$. Since $u \bar{u} \bar{v} v$ and $u x y v$, for $x \in N_{G}(u)$ and $y \in N_{G}(v)$, are shortest paths between $u$ and $v$, and $N_{G}(u) \cup N_{G}(v) \subseteq C$ we have that $N_{G}(u) \cup N_{G}(v) \cup\{\bar{u}, \bar{v}\} \subseteq I[u, v]$. Since $u y, v x \notin E(G)$, for $x \in N_{G}(u)$ and $y \in N_{G}(v)$, we have that $\bar{u} \bar{y}, \bar{v} \bar{x} \in E(\bar{G})$. Consequently $\bar{x} \in I[x, \bar{v}]$ and $\bar{y} \in I[y, \bar{u}]$. Therefore $N_{G}[\{u, v\}] \cup \overline{N_{G}[\{u, v\}]} \subseteq H(S)$.

Lemma 3.14. Let $G=(C \cup I, E)$ be a split autoconnected graph. Then $t\left(G^{\prime}\right)=1$ if and only if $t\left(\bar{G}^{\prime}\right)=1$.

Proof. Suppose that $t\left(G^{\prime}\right)=1$. Let $v$ be the trivial component of $G^{\prime}$. Since $N_{G}[v]=C, N_{\bar{G}}(\bar{v})=\bar{I}$, hence $\bar{I} \cup\{\bar{v}\}$ is a clique of $\bar{G}$. Since $\bar{C}$ is an independent set, at most one vertex from $\bar{C}$ can belong to a clique in $\bar{G}$. Hence $\bar{I} \cup\{\bar{v}\}$ is a maximum clique. Since $t\left(G^{\prime}\right)=1$, for every $u \in C \backslash\{v\},\left|N_{G}(u) \cap I\right| \geq 1$. Thus, for every $\bar{u} \in \bar{C} \backslash\{\bar{v}\},\left|N_{\bar{G}}(\bar{u})\right| \leq|\bar{I}|-1$. Then $\left|N_{\bar{G}}(\bar{u})\right|$ implies that $\bar{I} \cup\{\bar{v}\}$ is the unique maximum clique of $\bar{G}$.

Since $C$ is maximum, for every $y \in I$, we have that $\left|N_{G}(y)\right|<|C|$ and since $C$ is unique, there exists no $y \in I$ such that $y$ is adjacent to $|C|-1$ vertices. Then, for every $y \in I,\left|N_{G}(y)\right| \leq|C|-2$. This implies that, for every $\bar{y} \in \bar{I},\left|N_{\bar{G}}(\bar{y}) \cap \bar{C}\right| \geq 2$.

Since, for every $\bar{y} \in \bar{I}, N_{\bar{G}}(\bar{y}) \cap \bar{C} \neq \emptyset$, it follows that $\bar{v}$ is the unique trivial component of $\bar{G}^{\prime}$, therefore $t\left(\bar{G}^{\prime}\right)=1$.

Reciprocally, suppose that $t\left(\bar{G}^{\prime}\right)=1$. Since $\bar{G}$ is also split, by the same previous arguments, now applied for $\bar{G}^{\prime}$, we conclude that $t\left(G^{\prime}\right)=1$. 
Let $G$ be a split autoconnected graph. As we have done with the lower bounds, we establish a relation of the upper bounds of $h(G \bar{G})$ with the number of components of $G^{\prime}$. To show that we divided the proof concerning about the number of trivial and non-trivial components of $G^{\prime}$. The case of at least one of the parameters $n t\left(G^{\prime}\right), t\left(G^{\prime}\right), n t\left(\bar{G}^{\prime}\right)$ or $t\left(\bar{G}^{\prime}\right)$ be greater than one is provided by Theorem 3.15, and the remaining cases follow in Theorems 3.18 and 3.19 .

Theorem 3.15. Let $G=(C \cup I, E)$ be a split autoconnected graph. If $\max \left\{n t\left(G^{\prime}\right), t\left(G^{\prime}\right), n t\left(\bar{G}^{\prime}\right), t\left(\bar{G}^{\prime}\right)\right\} \geq 2$, then $h(G \bar{G}) \leq \max \left\{c\left(G^{\prime}\right), c\left(\bar{G}^{\prime}\right)\right\}$.

Proof. To proceed with the proof we consider two cases (1) nt $\left(G^{\prime}\right) \geq 2$ or $n t\left(\bar{G}^{\prime}\right) \geq 2$, and $(2) t\left(G^{\prime}\right) \geq 2$ or $t\left(\bar{G}^{\prime}\right) \geq 2$. By definition of complementary prism, we know that $G \bar{G}$ is isomorphic to $\bar{G} G$. Then, without loss of generality, we may consider $c\left(G^{\prime}\right) \geq c\left(\bar{G}^{\prime}\right)$. In view of that, we show a hull set of $G \bar{G}$ in each case: (1) $n t\left(G^{\prime}\right) \geq 2$ and (2) $t\left(G^{\prime}\right) \geq 2$.

Case 1. $n t\left(G^{\prime}\right) \geq 2$.

Let $\mathfrak{I}_{1}=\left\{i \in\left[c\left(G^{\prime}\right)\right]:\left|V\left(G_{i}\right)\right|=1\right\}$ and $\mathfrak{I}_{2}=\left[c\left(G^{\prime}\right)\right] \backslash \mathfrak{I}_{1}$.

For every $k \in\left[c\left(G^{\prime}\right)\right]$, we obtain the set $S$ by choosing $u_{k} \in V\left(G_{k}\right)$ such that $V\left(G_{k}\right)=\left\{u_{k}\right\}$ if $k \in \Im_{1}$ and $u_{k} \in V\left(G_{k}\right) \backslash C$ if $k \in \mathfrak{I}_{2}$. Notice that $|S|=c\left(G^{\prime}\right)$. We show that $H(S)=V(G \bar{G})$.

By hypothesis, $\left|\mathfrak{I}_{2}\right| \geq 2$. For all $i, j \in \mathfrak{I}_{2}, i \neq j$, since $d_{G}\left(u_{i}, u_{j}\right)=3$, Lemma 3.13 implies that $N_{G}\left[\left\{u_{i}, u_{j}\right\}\right] \cup$ $\overline{N_{G}\left[\left\{u_{i}, u_{j}\right\}\right]} \subseteq H(S)$.

Let $u \in N_{G}\left(u_{i}\right), u^{\prime} \in N_{G}\left(u_{j}\right)$, for some $i, j \in \mathfrak{I}_{2}, A=I \backslash N_{G}(u)$ and $B=I \backslash N_{G}\left(u^{\prime}\right)$. We have that $\bar{A} \subseteq N_{\bar{G}}(\bar{u})$, then $\bar{A} \subseteq I\left[\bar{u}, \bar{u}_{i}\right]$. Similarly, $\bar{B} \subseteq N_{\bar{G}}\left(\bar{u}^{\prime}\right)$, then $\bar{B} \subseteq I\left[\bar{u}^{\prime}, \bar{u}_{j}\right]$. Since $A \cup B=I, \bar{I} \subseteq H(S)$.

Only vertices of $I$ that are not yet in $H(S)$ are those who are at distance 2 to all the other vertices from $I$. However such vertices cannot exists because $n t\left(G^{\prime}\right) \geq 2$. Hence $I \subseteq H(S)$, which implies that $C \subseteq H(G)$ and finally, $\bar{C} \subseteq H(S)$. Therefore $S$ is a hull set of $G \bar{G}$, and $h(G \bar{G}) \leq c\left(G^{\prime}\right)$.

Case 2. $t\left(G^{\prime}\right) \geq 2$.

If $n t\left(G^{\prime}\right) \geq 2$ we fall back on Case 1. Thus, consider that $n t\left(G^{\prime}\right)=1$. Let $G_{1}$ be the non-trivial component of $G^{\prime}$ and $G_{i}$, for $i \in\left[c\left(G^{\prime}\right)\right] \backslash\{1\}$, be the trivial components of $G^{\prime}$. For some $x \in V\left(G_{1}\right) \cap C$, let $S=V\left(\overline{G_{i}}\right) \cup\{x\}$, for every $i \in\left[c\left(G^{\prime}\right)\right] \backslash\{1\}$. Notice that $|S|=c\left(G^{\prime}\right)$. We show that $S$ is a hull set of $G \bar{G}$.

By hypothesis, $t\left(G^{\prime}\right) \geq 2$. Then there exist $i, j \in\left[c\left(G^{\prime}\right)\right] \backslash\{1\}$ such that $G_{i}$ and $G_{j}$ are trivial components in $G^{\prime}$. Let $v_{i}$ (resp. $v_{j}$ ) be the single vertex in $V\left(G_{i}\right)$ (resp. $V\left(G_{j}\right)$ ). Since $v_{i} v_{j} \in E(G), \bar{v}_{i} \bar{v}_{j} \notin E(\bar{G})$. Consequently, $\bar{v}_{i} \bar{y} \bar{v}_{j}$ is a shortest $\left(\bar{v}_{i}, \bar{v}_{j}\right)$-path, for every $\bar{y} \in \bar{I}$. Then $\bar{I} \subseteq I\left[\bar{v}_{i}, \bar{v}_{j}\right]$.

For every $i \in\left[c\left(G^{\prime}\right)\right] \backslash\{1\}, d_{G}\left(x, v_{i}\right)=1$, then $v_{i} \in I\left[x, \bar{v}_{i}\right]$. Furthermore, vertices in $y \in I$ and $w \in$ $C \backslash\left\{x, v_{2}, \ldots, v_{c\left(G^{\prime}\right)}\right\}$ belong subsequently to $H(S)$, by the closed intervals $y \in I[x, \bar{y}]$ and $w \in I\left[y, v_{2}\right]$. Hence $V(G) \subseteq H(S)$.

Given that $V(G) \cup \bar{I} \subseteq H(S)$ it is easy to see that $V(\bar{G}) \subseteq H(S)$.

By all cases there exists a hull set of $G \bar{G}$ of order $c\left(G^{\prime}\right)$. Since $c\left(G^{\prime}\right) \geq c\left(\bar{G}^{\prime}\right)$, the result $h(G \bar{G}) \leq$ $\max \left\{c\left(G^{\prime}\right), c\left(\bar{G}^{\prime}\right)\right\}$ holds.

By Theorems 3.12 and 3.15 we obtain the equality stated in Corollary 3.16. That result evidences that for complemetary prisms of split graphs the hull number can be unlimited. The class of complementary prisms $K_{n} \bar{K}_{n} \bar{K}_{n} \bar{K}_{n}$ is an example of $h\left(K_{n} \bar{K}_{n} \bar{K}_{n} \bar{K}_{n}\right)=n$.

Corollary 3.16. Let $G=(C \cup I, E)$ be a split autoconnected graph. If $\max \left\{n t\left(G^{\prime}\right), t\left(G^{\prime}\right), n t\left(\bar{G}^{\prime}\right), t\left(\bar{G}^{\prime}\right)\right\} \geq 2$, then $h(G \bar{G})=\max \left\{c\left(G^{\prime}\right), c\left(\bar{G}^{\prime}\right)\right\}$.

Proof. It follows directly from Theorems 3.12 and 3.15 . 
Now, it remains the cases in which $n t\left(G^{\prime}\right)<2, n t\left(\bar{G}^{\prime}\right)<2, t\left(G^{\prime}\right)<2$ and $t\left(\bar{G}^{\prime}\right)<2$. Since we disregard the trivial graphs, we have that $n t\left(G^{\prime}\right)=1$ and $n t\left(\bar{G}^{\prime}\right)=1$. By Lemma 3.14 if $t\left(G^{\prime}\right)=1$, then $t\left(\bar{G}^{\prime}\right)=1$. Otherwise, for $t\left(G^{\prime}\right) \neq 1$, we have that $t\left(\bar{G}^{\prime}\right)=0$ or $t\left(\bar{G}^{\prime}\right) \geq 2$. If $t\left(\bar{G}^{\prime}\right) \geq 2$ the proof follows by Theorem 3.15 Case 2 . So, it remains the cases $t\left(G^{\prime}\right)=0, t\left(\bar{G}^{\prime}\right)=0$, and $t\left(G^{\prime}\right)=1, t\left(\bar{G}^{\prime}\right)=1$, that follows in Theorems 3.18 and 3.19, respectively.

We first show a proposition that will be useful in the proof of Theorem 3.18. The next proposition is a slight modification of Proposition 3.4 now considering a split graph $G=(C \cup I, E)$. For a set of vertices $S$ of $G \bar{G}$, Proposition 3.17 shows that every vertex lying on a path in the component graph $G^{\prime}$ of $G$ will belong to $H(S)$ depending if some specific vertices in $C \cup \bar{I}$ belong to $H(S)$.

Proposition 3.17. Let $G=(C \cup I, E)$ be a split autoconnected graph. For $k \geq 2$, let $w_{0} v_{1} w_{1} \ldots v_{k} w_{k}$ (or $\left.w_{0} v_{1} w_{1} \ldots v_{k}\right)$ be a path in $G^{\prime}$, such that $v_{i} \in I$, for every $i \in[k]$, and $w_{j} \in C$, for every $j \in[k] \cup\{0\}$. Consider that, for every $i \in[k]$, there exists $z_{i} \in C$ such that $v_{i} z_{i} \notin E(G)$, and let $S \subseteq V(G \bar{G})$. If $\left\{w_{0}, z_{1}, \ldots z_{k}, \bar{v}_{1}, \ldots, \bar{v}_{k}\right\} \subseteq H(S)$, then $w_{k} \in H(S)$ (resp. $v_{k} \in H(S)$ ).

Proof. The proof is by induction on $k$. Let $k=1$. Since $w_{0}, \bar{v}_{1} \in H(S)$, and $v_{1} \bar{v}_{1} \in E(G \bar{G})$, we obtain that $v_{1} \in I\left[w_{0}, \bar{v}_{1}\right]$. Since $z_{i} \in H(S)$, and $v_{1} z_{1} \notin E(G)$, then $w_{1} \in I\left[v_{1}, z_{1}\right]$.

Let $k>1$. By induction hypothesis, $w_{k-1} \in H(S)$. Then, since $\bar{v}_{k} \in H(S)$, we have that $v_{k} \in I\left[w_{k-1}, \bar{v}_{k}\right]$. Since $z_{k} \in H(S)$, and $v_{k} z_{k} \notin E(G)$, it follows that $w_{k} \in I\left[v_{k}, z_{k}\right]$.

Theorem 3.18. Let $G=(C \cup I, E)$ be a split autoconnected graph such that $n t\left(G^{\prime}\right)=1, n t\left(\bar{G}^{\prime}\right)=1, t\left(G^{\prime}\right)=0$ and $t\left(\bar{G}^{\prime}\right)=0$. Then $h(G \bar{G}) \leq 3$.

Proof. To prove that $h(G \bar{G}) \leq 3$ we must construct a hull set of $G \bar{G}$ of order three. We first show the existence of three vertices that are the basis of our construction. Consider $d=\max \left\{\operatorname{deg}_{G}(v): v \in I\right\}$, and $y \in I$ such that $\operatorname{deg}_{G}(y)=d$.

Claim 1. There exist $x \in C \backslash N_{G}(y)$, and $y^{\prime} \in N_{G}(x) \cap I$.

Proof of Claim 1. Suppose, by contradiction, that there exists no $x \in C \backslash N_{G}(y)$. Then, for every $x \in C$, $x \in N_{G}(y)$. This implies that $C \cup\{y\}$ is a clique of $G$, a contradiction, since we consider a partition of $V(G)$ such that $C$ is a maximum clique. Now, since $t\left(G^{\prime}\right)=0$ and $x y \notin E(G), x$ is not an isolated vertex in $G^{\prime}$, then there exists $y^{\prime} \in N_{G}(x) \cap I$.

Let $x \in C \backslash N_{G}(y)$, and $y^{\prime} \in N_{G}(x) \cap I$. To avoid repetitive notation when considering the operations of union, intersection and symmetric difference on the sets $N_{G}(y)$ and $N_{G}\left(y^{\prime}\right)$, we write $U=N_{G}(y) \cup N_{G}\left(y^{\prime}\right)$, $A=N_{G}(y) \cap N_{G}\left(y^{\prime}\right)$, and $D=N_{G}(y) \triangle N_{G}\left(y^{\prime}\right)$, respectively.

We consider a partition $R \cup L$ of $I \backslash\left\{y, y^{\prime}\right\}$ such that $R=\left\{v \in I \backslash\left\{y, y^{\prime}\right\}: \forall u \in D, u v \in E(G)\right\}$, and $L=I \backslash\left(R \cup\left\{y, y^{\prime}\right\}\right)$. Furthermore, we let $R_{1}=\left\{v \in R: N_{G}(v) \cap(C \backslash U) \neq \emptyset\right\}$, and $R_{2}=R \backslash R_{1}$. We show hull sets of $G \bar{G}$ considering $R_{1}$ be empty or not.

Case 1. $R_{1}=\emptyset$.

Let $S=\left\{x, y, \bar{y}^{\prime}\right\}$. See Figure 4 for an example. First, we have that $N_{G}(y) \subseteq I[x, y], y^{\prime} \in I\left[x, \bar{y}^{\prime}\right]$ and $\bar{y} \in I\left[y, \bar{y}^{\prime}\right]$.

Since $\operatorname{deg}_{G}(y)$ is maximum in $I$, there exists $z \in N_{G}(y)$ such that $y^{\prime} z \notin E(G)$. Thus $N_{G}\left(y^{\prime}\right) \subseteq I\left[y^{\prime}, z\right]$, which implies that $U \subseteq H(S)$. Since $D \subseteq U \subseteq H(S)$, and, for every $w \in N_{G}\left(y^{\prime}\right) \backslash A, \bar{w} \bar{y} \in E(\bar{G})$ (resp. for every $\left.w^{\prime} \in N_{G}(y) \backslash A \bar{w}^{\prime} \bar{y}^{\prime} \in E(\bar{G})\right)$, we obtain that $\bar{D} \subseteq I\left[D \cup\left\{\bar{y}, \bar{y}^{\prime}\right\}\right]$.

Let $v \in L$. By definition of $L$, there exists $z \in D$ such that $v z \notin E(G)$, which implies that $\bar{v} \bar{z} \in E(\bar{G})$. Since $\bar{z}$ is either adjacent to $\bar{y}$ or $\bar{y}^{\prime}$, and, for every $\bar{v} \in \bar{L}, \bar{v} \bar{y}, \bar{v} \bar{y}^{\prime} \in E(\bar{G})$, it follows that $\bar{v} \in I\left[\left\{\bar{y}, \bar{y}^{\prime}, \bar{z}\right\}\right]$. Hence $\bar{L} \subseteq H(S)$. By definition of $L$ we have also that $\bar{L} \cup\left\{\bar{y}, \bar{y}^{\prime}\right\}=\bar{I} \backslash \bar{R}_{2} \subseteq H(S)$.

Now, we show that $L \cup\left(N_{G}(L) \backslash U\right) \subseteq H(S)$. For that, we are interested in the paths of minimum length between $L \cup\left(N_{G}(L) \backslash U\right)$ and $U$. 

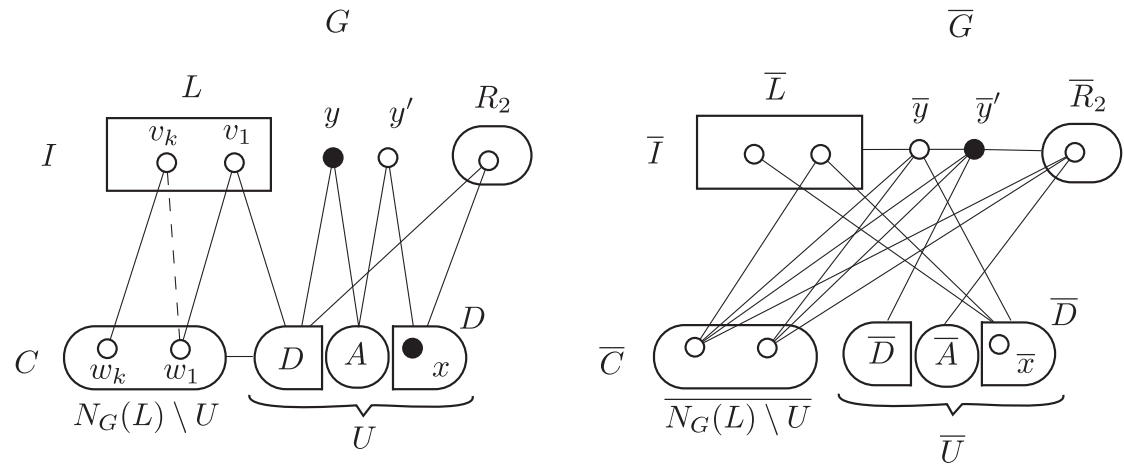

FiguRE 4. Example of hull set for Case 1 of Theorem 3.18.

Since $G^{\prime}$ is connected, for $k \geq 2$, there exists a shortest path $w_{0} v_{1} w_{1} \ldots v_{k} w_{k}$ (or $w_{0} v_{1} w_{1} \ldots v_{k}$ ) in $G^{\prime}$ between $w_{0} \in U$ and $w_{k} \in N_{G}(L) \backslash U$ (resp. $v_{k} \in L$ ), where $v_{i} \in I, w_{i} \in C$, for every $i \in[k]$.

Recall that, up to this point $\left(R_{2} \cup \bar{R}_{2}\right) \cap H(S)=\emptyset$. By definition of $R_{2}, N_{G}\left(R_{2}\right) \subseteq U$. Then the shortest path $w_{0} v_{1} w_{1} \ldots v_{k} w_{k}$ (or $w_{0} v_{1} w_{1} \ldots v_{k}$ ) in $G^{\prime}$ does not contain vertices from $R_{2}$. From this observation we can proceed to show that $v_{1}, w_{1}, \ldots, v_{k}, w_{k}$ will be successively included to $H(S)$.

By the maximality of $\operatorname{deg}_{G}(y)$ we have that $\operatorname{deg}_{G}\left(v_{i}\right)<\operatorname{deg}_{G}(y)+1=\left|N_{G}(y) \cup\{x\}\right|$, for every $i \in[k]$. Hence, there exists $z_{i} \in N_{G}(y) \cup\{x\}$ such that $v_{i} z_{i} \notin E(G)$ for every $i \in[k]$. Since $w_{0}, z_{1}, \ldots, z_{k} \in U \subseteq H(S)$, and $\bar{v}_{i} \in \bar{I} \backslash \bar{R}_{2} \subseteq H(S)$, for every $i \in[k]$, it follows from Proposition 3.17 that $w_{k} \in H(S)$ (resp. $v_{k} \in H(S)$ ). Consequently $L \cup\left(N_{G}(L) \backslash U\right) \subseteq H(S)$.

It remains to show that $R_{2} \cup \bar{R}_{2} \cup \bar{A} \cup \overline{N_{G}(L) \backslash U} \subseteq H(S)$.

Since $\bar{G}^{\prime}$ is connected, for $k \geq 2$, there exists a shortest path $\bar{w}_{0} \bar{v}_{1} \bar{w}_{1} \ldots \bar{v}_{k} \bar{w}_{k}\left(\right.$ or $\left.\bar{w}_{0} \bar{v}_{1} \bar{w}_{1} \ldots \bar{v}_{k}\right)$ in $\bar{G}^{\prime}$ between $\bar{w}_{0} \in \bar{I} \backslash \bar{R}_{2}$ and $\bar{w}_{k} \in \bar{R}_{2}$ (resp. $\left.\bar{v}_{k} \in \bar{A} \cup \bar{N}_{G}(L) \backslash U\right)$, where $\bar{v}_{i} \in \bar{C}$, and $\bar{w}_{i} \in \bar{I}$, for every $i \in[k]$.

We show that, for every $i \in[k]$, there exists $\bar{z} \in \bar{I} \backslash \bar{R}_{2}$ such that $\bar{v}_{i} \bar{z} \notin E(\bar{G})$. It is clear that $\bar{z}=\bar{y}$ for every $\bar{v} \in \bar{A}$, since $\bar{v} \bar{y} \notin E(\bar{G})$. Now, let $\bar{v} \in \overline{N_{G}(L) \backslash U}$. We know that $N_{G}\left(R_{2} \cup\left\{y, y^{\prime}\right\}\right) \cap\left(N_{G}(L) \backslash U\right)=\emptyset$. Since $G^{\prime}$ is connected, there exists $z \in L$ such that $v z \in E(G)$, which implies that $\bar{v} \bar{z} \notin E(\bar{G})$, with $\bar{z} \in \bar{L} \subseteq \bar{I} \backslash \bar{R}_{2}$.

Since $\bar{w}_{0}, \bar{z} \in \bar{I} \backslash \bar{R}_{2} \subseteq H(S)$, and $v_{i} \in A \cup N_{G}(L) \subseteq H(S)$, for every $i \in[k]$, Proposition 3.17 (dual) implies that $\bar{w}_{k} \in H(S)$ (resp. $\bar{v}_{k} \in H(S)$ ). Hence, $\bar{R}_{2} \cup \bar{A} \cup \overline{N_{G}(L) \backslash U} \subseteq H(S)$. Finally, since $D \cup \bar{R}_{2} \subseteq H(S)$, we obtain that $R_{2} \subseteq I\left[D \cup \bar{R}_{2}\right]$, which completes the proof of Case 1 .

Case 2. $R_{1} \neq \emptyset$.

Let $y^{\prime \prime} \in R_{1}$ such that $N_{G}\left(y^{\prime \prime}\right) \backslash U$ is maximum, and $S=\left\{x, y, \bar{y}^{\prime \prime}\right\}$. First, we have that $N_{G}(y) \subseteq I[x, y]$, $y^{\prime \prime} \in I\left[x, \bar{y}^{\prime \prime}\right]$, and $\bar{y} \in I\left[y, \bar{y}^{\prime \prime}\right]$. Since $\operatorname{deg}_{G}(y)$ is maximum, $y^{\prime \prime}$ is not adjacent to every vertex in $N_{G}(y) \cup\{x\}$, then $N_{G}\left(y^{\prime \prime}\right) \subseteq I\left[N_{G}(y) \cup\left\{x, y^{\prime \prime}\right\}\right]$.

Let $x^{\prime \prime} \in N_{G}\left(y^{\prime \prime}\right) \backslash U$. Since $x^{\prime \prime} \in H(S)$, and $\bar{x}^{\prime \prime} \bar{y} \in E(\bar{G})$, we obtain that $\bar{x}^{\prime \prime} \in I\left[x^{\prime \prime}, \bar{y}\right]$. Hence $\overline{N_{G}\left(y^{\prime \prime}\right) \backslash U \subseteq}$ $H(S)$. Since $\bar{x}^{\prime \prime} \bar{y}^{\prime} \in E(\bar{G})$, and $\bar{x}^{\prime \prime} \bar{y}^{\prime \prime} \notin E(\bar{G})$, it follows that $\bar{y}^{\prime} \in I\left[\bar{x}^{\prime \prime}, \bar{y}^{\prime \prime}\right]$. Consequently $y^{\prime} \in I\left[x, \bar{y}^{\prime}\right]$. This implies that $\bar{D} \in I\left[D \cup\left\{\bar{y}, \bar{y}^{\prime}\right\}\right]$. Since $x^{\prime \prime}$ is not adjacent to any vertex in $R_{2}$, we have that $\bar{x}^{\prime \prime}$ is adjacent to every vertex in $\bar{R}_{2}$. Thus, $\bar{R}_{2} \subseteq I\left[\bar{x}^{\prime \prime}, \bar{y}^{\prime \prime}\right]$. Then, $R_{2} \subseteq I\left[D \cup \bar{R}_{2}\right]$.

Let $v \in L$. By definition of $L$, there exists $z \in D$ such that $v z \notin E(G)$, which implies that $\bar{v} \bar{z} \in E(\bar{G})$. Since $\bar{z}$ is either adjacent to $\bar{y}$ or $\bar{y}^{\prime}$, and, for every $\bar{v} \in \bar{L}, \bar{v} y, \bar{v} \bar{y}^{\prime} \in E(\bar{G})$, it follows that $\bar{v} \in I\left[\left\{\bar{y}, \bar{y}^{\prime}, \bar{z}\right\}\right]$. Hence $\bar{L} \subseteq H(S)$.

We consider $X \cup Y$ a partition of $R_{1} \backslash\left\{y^{\prime \prime}\right\}$ such that $X=\left\{v \in R_{1} \backslash\left\{y^{\prime \prime}\right\}: \forall u \in\left(A \backslash N_{G}\left(y^{\prime \prime}\right)\right) \cup\left(N_{G}\left(y^{\prime \prime}\right) \backslash U\right)\right.$, $u v \in E(G)\}$, and $Y=R_{1} \backslash\left(X \cup\left\{y^{\prime \prime}\right\}\right)$.

Let $a \in A \backslash N_{G}\left(y^{\prime \prime}\right)$. Since $\bar{a} \bar{y}^{\prime \prime} \in E(\bar{G})$, we obtain that $\bar{a} \in I\left[a, \bar{y}^{\prime \prime}\right]$. Thus, $\overline{A \backslash N_{G}\left(y^{\prime \prime}\right)} \subseteq H(S)$. Let $v \in Y$. By definition of $Y$, and since we selected $y^{\prime \prime} \in R_{1}$ such that $N_{G}\left(y^{\prime \prime}\right) \backslash U$ is maximum, there exists $u \in\left(N_{G}\left(y^{\prime \prime}\right) \backslash U\right)$ 
such that $u v \notin E(G)$, then $\bar{u} \bar{v} \in E(\bar{G})$. Since $\bar{u} \bar{y}^{\prime \prime} \notin E(\bar{G})$, and $\bar{u} \in \overline{N_{G}\left(y^{\prime \prime}\right) \backslash U} \subseteq H(S)$, we have that $\bar{v} \in I\left[\bar{u}, \bar{y}^{\prime \prime}\right]$. It follows that $\bar{Y} \subseteq H(S)$, and $Y \subseteq I[D \cup \bar{Y}]$. Hence, $N_{G}(Y) \subseteq H(S)$.

Now, we show that $L \cup\left(N_{G}(L) \backslash\left(N_{G}\left(Y \cup y^{\prime \prime}\right) \cup U\right)\right) \subseteq H(S)$.

Since $G^{\prime}$ is connected, for $k \geq 2$, there exists a shortest path $w_{0} v_{1} w_{1} \ldots v_{k} w_{k}$ (or $w_{0} v_{1} w_{1} \ldots v_{k}$ ) in $G^{\prime}$ between $w_{0} \in N_{G}\left(Y \cup y^{\prime \prime}\right) \cup U$ and $w_{k} \in N_{G}(L) \backslash\left(N_{G}\left(Y \cup y^{\prime \prime}\right) \cup U\right.$ ) (resp. $v_{k} \in L$ ), where $v_{i} \in I, w_{i} \in C$, for every $i \in[k]$.

By definition of $X$, no vertex in $X$ is adjacent to some vertex in $N_{G}(L) \backslash\left(N_{G}\left(Y \cup y^{\prime \prime}\right) \cup U\right)$, then $w_{0} v_{1} w_{1} \ldots v_{k} w_{k}$ (resp. $w_{0} v_{1} w_{1} \ldots v_{k}$ ) does not contain vertices from $X$.

By the maximality of $\operatorname{deg}_{G}(y)$ we have that $\operatorname{deg}_{G}\left(v_{i}\right)<\operatorname{deg}_{G}(y)+1=\left|N_{G}(y) \cup\{x\}\right|$, for every $i \in[k]$. Hence, there exists $z_{i} \in N_{G}(y) \cup\{x\}$ such that $v_{i} z_{i} \notin E(G)$, for every $i \in[k]$. Since $w_{0}, z_{1}, \ldots, z_{k} \in N_{G}\left(Y \cup y^{\prime \prime}\right) \cup U \subseteq$ $H(S)$, and $\bar{v}_{i} \in \bar{I} \backslash \bar{X} \subseteq H(S)$, for every $i \in[k]$, it follows from Proposition 3.17 that $w_{k} \in H(S)$ (resp. $\left.v_{k} \in H(S)\right)$. Consequently $L \cup\left(N_{G}(L) \backslash\left(N_{G}\left(Y \cup y^{\prime \prime}\right) \cup U\right)\right) \subseteq H(S)$.

Similarly to Case 1, since $\bar{G}^{\prime}$ is connected, Proposition 3.17 (dual) can be used to show that $\bar{X} \cup \overline{A \cap N_{G}\left(y^{\prime \prime}\right)} \cup$ $\overline{N_{G}(L) \backslash\left(N_{G}\left(Y \cup y^{\prime \prime}\right) \cup U\right)} \subseteq H(S)$. Since $\bar{X} \subseteq H(S)$, we obtain that $X \subseteq I[\bar{X} \cup U]$, therefore $H(S)=V(G \bar{G})$.

By all cases we have that there exists a hull set of $G \bar{G}$ of order three, which completes the proof.

Figure 4 shows an example of complementary prism $G \bar{G}$ illustrating Case 1 of Theorem 3.18. The black vertices represent a hull set of $G \bar{G}$.

Theorem 3.19. Let $G=(C \cup I, E)$ be a split autoconnected graph such that $n t\left(G^{\prime}\right)=1, n t\left(\bar{G}^{\prime}\right)=1, t\left(G^{\prime}\right)=1$ and $t\left(\bar{G}^{\prime}\right)=1$. Then $h(G \bar{G}) \leq 3$.

Proof. Let $v$ be the trivial component of $G^{\prime}$. Let $G_{1}$ be the graph induced by $V(G) \backslash\{v\}$.

Since $G_{1}$ is a subgraph of $G$ that satisfies $n t\left(G_{1}^{\prime}\right)=1, n t\left(\bar{G}_{1}^{\prime}\right)=1, t\left(G_{1}^{\prime}\right)=0$ and $t\left(\bar{G}_{1}^{\prime}\right)=0$, we proceed in a similar way as in the proof of Theorem 3.18. Consider $d=\max \left\{\operatorname{deg}_{G}(w): w \in I\right\}$, and $y \in I$ such that $\operatorname{deg}_{G}(y)=d$. Let $x \in C \backslash\left(N_{G}(y) \cup\{v\}\right)$, and $y^{\prime} \in N_{G}(x) \cap I$. Claim 1 implies the existence of $x, y, y^{\prime}$ in $V\left(G_{1}\right) \subseteq V(G)$.

Let $U=N_{G}(y) \cup N_{G}\left(y^{\prime}\right), A=N_{G}(y) \cap N_{G}\left(y^{\prime}\right)$, and $D=N_{G}(y) \triangle N_{G}\left(y^{\prime}\right)$. Consider $R=\left\{v \in I \backslash\left\{y, y^{\prime}\right\}\right.$ : $\forall u \in D, u v \in E(G)\}$, and $R_{1}=\left\{v \in R: N_{G}(v) \cap(C \backslash U) \neq \emptyset\right\}$. We show hull sets of $G \bar{G}$ considering $R_{1}$ be empty or not.

Case 1. $R_{1}=\emptyset$.

Let $S=\left\{y, y^{\prime}, \bar{v}\right\}$. We have that $A=N_{G}(y) \cap N_{G}\left(y^{\prime}\right) \subseteq I\left[y, y^{\prime}\right]$. Since $n t\left(G^{\prime}\right)=1$, we have that $d_{G}\left(y, y^{\prime}\right)<3$, hence $A \neq \emptyset$. Since $v$ is adjacent to every vertex in $A$, we obtain that $v \in I[A \cup\{\bar{v}\}]$. Consequently $U=N_{G}(y) \cup N_{G}\left(y^{\prime}\right) \subseteq I\left[\left\{v, y, y^{\prime}\right\}\right]$. Since $v y, v y^{\prime} \notin E(G), \bar{v} \bar{y}, \bar{v} \bar{y}^{\prime} \in E(\bar{G})$, then $\bar{y}, \bar{y}^{\prime} \in I\left[\left\{y, y^{\prime}, \bar{v}\right\}\right]$.

At this point, we have that $x, y, \bar{y}^{\prime} \in H(S)$. Since $G_{1}$ is a subgraph of $G$ with $n t\left(G_{1}^{\prime}\right)=1, n t\left(\bar{G}_{1}^{\prime}\right)=1$, $t\left(G_{1}^{\prime}\right)=0$ and $t\left(\bar{G}_{1}^{\prime}\right)=0$, Theorem 3.18 Case 1 implies that $V\left(G_{1}\right) \cup V\left(\bar{G}_{1}\right) \subseteq H_{G_{1} \bar{G}_{1}}\left(\left\{x, y, \bar{y}^{\prime}\right\}\right)$. Since $G_{1} \bar{G}_{1}$ is an isometric subgraph of $G \bar{G}$, Lemma 3.2 implies that $V\left(G_{1}\right) \cup V\left(\bar{G}_{1}\right) \subseteq H\left(\left\{x, y, \bar{y}^{\prime}\right\}\right)$, consequently $V(G \bar{G})=H(S)$.

Case 2. $R_{1} \neq \emptyset$.

Let $y^{\prime \prime} \in R_{1}$ such that $N_{G}\left(y^{\prime \prime}\right) \backslash U$ is maximum, and $S=\left\{y, y^{\prime \prime}, \bar{v}\right\}$. We have that $N_{G}(y) \cap N_{G}\left(y^{\prime \prime}\right) \subseteq I\left[y, y^{\prime \prime}\right]$. Since $n t\left(G^{\prime}\right)=1$, we have that $d_{G}\left(y, y^{\prime \prime}\right)<3$, hence $N_{G}(y) \cap N_{G}\left(y^{\prime \prime}\right) \neq \emptyset$. Let $z \in N_{G}(y) \cap N_{G}\left(y^{\prime \prime}\right)$. Since $v z \in E(G)$, we have that $v \in I[z, \bar{v}]$. It follows that $N_{G}(y) \cup N_{G}\left(y^{\prime \prime}\right) \subseteq I\left[\left\{v, y, y^{\prime \prime}\right\}\right]$. Since $v y, v y^{\prime \prime} \notin E(G)$, $\bar{v} \bar{y}, \bar{v} \bar{y}^{\prime \prime} \in E(\bar{G})$, then $\bar{y}, \bar{y}^{\prime \prime} \in I\left[\left\{y, y^{\prime \prime}, \bar{v}\right\}\right]$.

So far, we have that $x, y, \bar{y}^{\prime \prime} \in H(S)$. Since $G_{1}$ is a subgraph of $G$ with $n t\left(G_{1}^{\prime}\right)=1, n t\left(\bar{G}_{1}^{\prime}\right)=1, t\left(G_{1}^{\prime}\right)=0$ and $t\left(\bar{G}_{1}^{\prime}\right)=0$, Theorem 3.18 Case 2 implies that $V\left(G_{1}\right) \cup V\left(\bar{G}_{1}\right) \subseteq H_{G_{1} \bar{G}_{1}}\left(\left\{x, y, \bar{y}^{\prime \prime}\right\}\right)$. Since $G_{1} \bar{G}_{1}$ is an isometric subgraph of $G \bar{G}$, Lemma 3.2 implies that $V\left(G_{1}\right) \cup V\left(\bar{G}_{1}\right) \subseteq H\left(\left\{x, y, \bar{y}^{\prime \prime}\right\}\right)$, consequently $V(G \bar{G})=H(S)$.

By all cases we have that $S$ is a hull set of $G \bar{G}$, and the result $h(G \bar{G}) \leq 3$ holds. 
Still considering a split autoconnected graph $G$, we show in Corollary 3.20 an equality for the case $n t\left(G^{\prime}\right)=1$, $n t\left(\bar{G}^{\prime}\right)=1, t\left(G^{\prime}\right)=1$, and $t\left(\bar{G}^{\prime}\right)=1$.

Corollary 3.20. Let $G=(C \cup I, E)$ be a split autoconnected graph. If $n t\left(G^{\prime}\right)=n t\left(\bar{G}^{\prime}\right)=t\left(G^{\prime}\right)=t\left(\bar{G}^{\prime}\right)=1$, then $h(G \bar{G})=3$.

Proof. Let $v$ be the trivial component of $G^{\prime}$. The upper bound follows by Theorem 3.19. So, remains to show the lower bound $h(G \bar{G}) \geq 3$.

Since $v$ is a simplicial vertex in $G$, and $\bar{v}$ is a simplicial vertex in $\bar{G}$, Lemma 3.3 implies that $S \cap\{v, \bar{v}\} \neq \emptyset$. Let $x \in\{v, \bar{v}\}$, and suppose that $S=\{u, x\}$ is a hull set of $G \bar{G}$. Since $d_{G \bar{G}}(u, x)<2$, for every $u \in V(G \bar{G}) \backslash\{v, \bar{v}\}$, we have that $H(S)=N_{G \bar{G}}[u] \cap N_{G \bar{G}}[x]$. This implies that $H(S) \neq V(G \bar{G})$, a contradiction. Therefore, $h(G \bar{G}) \geq 3$.

We close our contributions with the result expressed in Corollary 3.21. We consider split autoconnected graphs $G$ such that $\operatorname{diam}(G)=\operatorname{diam}(\bar{G})=2$. If $n t\left(G^{\prime}\right) \geq 2$ or $n t\left(\bar{G}^{\prime}\right) \geq 2$, then $\operatorname{diam}(G)=3$ or $\operatorname{diam}(\bar{G})=3$, which does not belong to the current case. If $t\left(G^{\prime}\right) \geq 2$ or $t\left(\bar{G}^{\prime}\right) \geq 2$ we fall back on Theorem 3.15, hence $h(G \bar{G})=\max \left\{c\left(G^{\prime}\right), c\left(\bar{G}^{\prime}\right)\right\}$. Thus, we consider $n t\left(G^{\prime}\right)=1, n t\left(\bar{G}^{\prime}\right)=1, t\left(G^{\prime}\right) \leq 1$, and $t\left(\bar{G}^{\prime}\right) \leq 1$.

Corollary 3.21. Let $G=(C \cup I, E)$ be a split autoconnected graph such that $\operatorname{diam}(G)=\operatorname{diam}(\bar{G})=2$. If $\max \left\{n t\left(G^{\prime}\right), t\left(G^{\prime}\right), n t\left(\bar{G}^{\prime}\right), t\left(G^{\prime}\right)\right\}<2$, then $h(G \bar{G})=3$.

Proof. It follows from Theorems 3.6 and 3.15 .

Acknowledgements. This study was financed in part by the Coordenação de Aperfeiçoamento de Pessoal de Nível Superior - Brasil (CAPES) - Finance Code 001.

\section{REFERENCES}

[1] M. Albenque and K. Knauer, Convexity in partial cubes: the hull number. In: LATIN 2014: Theoretical Informatics. Springer, New York, NY (2014) 421-432.

[2] B.S. Anand, M. Changat, S. Klavžar and I. Peterin, Convex sets in lexicographic products of graphs. Graphs Comb. 28 (2012) $77-84$.

[3] J. Araujo, V. Campos, F. Giroire, N. Nisse, L. Sampaio and R. Soares, On the hull number of some graph classes. Theor. Comput. Sci. 475 (2013) 1-12.

[4] S.R. Canoy, Jr. and I. Garces, Convex sets under some graph operations. Graphs Comb. 18 (2002) $787-793$.

[5] C.C. Centeno, M.C. Dourado, L.D. Penso, D. Rautenbach and J.L. Szwarcfiter, Irreversible conversion of graphs. Theor. Comput. Sci. 412 (2011) 3693-3700.

[6] E.M.M. Coelho, H. Coelho, J.R. Nascimento and J.L. Szwarcfiter, On the geodetic hull number of complementary prisms. Preprint: arXiv: 1807.08295 (2018).

[7] P. Domingos and M. Richardson, Mining the network value of customers. In: Proceedings of the Seventh ACM SIGKDD International Conference on Knowledge Discovery and Data Mining. KDD '01. ACM. New York, NY, (2001) 57-66.

[8] M.C. Dourado and R.M. Sampaio, Complexity aspects of the triangle path convexity. Discrete Appl. Math. 206 (2016) 39-47.

[9] M.C. Dourado, J.G. Gimbel, J. Kratochvíl, F. Protti and J.L. Szwarcfiter, On the computation of the hull number of a graph. Discrete Math. 309 (2009) 5668-5674.

[10] M.C. Dourado, F. Protti, D. Rautenbach and J.L. Szwarcfiter, On the hull number of triangle-free graphs. SIAM J. Discrete Math. 23 (2010) 2163-2172.

[11] M.C. Dourado, F. Protti and J.L. Szwarcfiter, Complexity results related to monophonic convexity. Discrete Appl. Math. 158 (2010) 1268-1274.

[12] M.C. Dourado, L.D. Penso and D. Rautenbach, On the geodetic hull number of $P_{k}$-free graphs. Theor. Comput. Sci. 640 (2016) 52-60.

[13] P.A. Dreyer and F.S. Roberts, Irreversible $k$-threshold processes: graph-theoretical threshold models of the spread of disease and of opinion. Discrete App. Math. 157 (2009) 1615-1627.

[14] M.A. Duarte, L. Penso, D. Rautenbach and U. dos Santos Souza, Complexity properties of complementary prisms. J. Comb. Optim. 33 (2017) 365-372.

[15] M.G. Everett and S.B. Seidman, The hull number of a graph. Discrete Math. 57 (1985) 217-223. 
[16] S. Foldes and P.L. Hammer, Split graphs. In: Proceedings 8th Southeastern Conference on Combinatorics, Graph Theory and Computing, Louisiana State University, Baton Rouge, LA (1977) 311-315.

[17] T.W. Haynes, M.A. Henning, P.J. Slater and L.C. van der Merwe, The complementary product of two graphs. Bull. Inst. Comb. App. 51 (2007) 21-30.

[18] C. Hernando, T. Jiang, M. Mora, I.M. Pelayo and C. Seara, On the Steiner, geodetic and hull numbers of graphs. Discrete Math. 293 (2005) 139-154.

[19] M.M. Kanté and L. Nourine, Polynomial time algorithms for computing a minimum hull set in distance-hereditary and chordal graphs. SIAM J. Discrete Math. 30 (2016) 311-326.

[20] D. Peleg, Local majorities, coalitions and monopolies in graphs: a review. Theor. Comput. Sci. 282 (2002) $231-257$.

[21] I. Peterin, Intervals and convex sets in strong product of graphs. Graphs Comb. 29 (2013) 705-714. 